\title{
Las relaciones China - Rusia en la era de la Iniciativa de la Franja y la Ruta: la mutua adaptación a la asimetría
}

\author{
China-Russia relations in the age of the Belt and Road \\ INITIATIVE: Mutual adaptation to asymmetry
}

\section{Relações China - Rússia na era do INICIATIVA do Cinturão e Rota: adaptação mútua à assimetria}

1. Rubén Ruiz Ramas es profesor en el Departamento de Ciencias Políticas y de la Administración de la UNED (Madrid, España). En la actualidad es vicedecano de Relaciones Internacionales y Erasmus de la Facultad de Ciencias Políticas y Sociología de la UNED. Anteriormente hasido investigadorsenior en la EscueladeEstudios Internacionales dela Sun Yat-sen University (Cantón, China), e investigador asociado en el Departamento de Política Internacional de la City University of London. Ruiz-Ramas es Licenciado en Historia porla Universidad de Zaragoza, Diploma en Estudios Políticos y Constitucionales en el Centro de Estudios Políticos y Constitucionales (CEPC), Diploma de Estudios Avanzados en Ciencia Política dentro del programa "Procesos políticos en Europa del Este y Antigua Unión Soviética" de la UNED, y finalmente, doctor en Ciencias Políticas y de la Administración (Premio Extraordinario de Doctorado). https://orcid. org/0000-0003-1100-1132 (iD)

\author{
Rubén Ruiz-Ramas ${ }^{1}$
}

DOI: 10.5752/P.2317-773X.2020v8.n3.p28

Recibido el 29 de noviembre de 2019

Aprobado el 30 de marzo de 2020

\section{RESUMEN}

El artículo analiza la naturaleza y alcance de las relaciones entre China y Rusia en la era de la Franjay la Ruta,coincidenteen el tiempo con el lanzamiento de la Unión Económica Euroasiática. Las principales conclusiones del estudio son tres. Por un lado, la voluntad de hacer progresar la "asociación estratégica" de gobernantes chinos y rusos, y en paralelo, de minimizar las fuentes de conflicto, han devenido en un avance sustan cial de su cooperación, aplicable tanto a acuerdos bilaterales como regionales. Por otro lado, persiste un fuerte desequilibrio entre el contenido de estos acuerdos y la retórica con que, especialmente Rusia, proyecta sus relaciones con China. La tercera conclusión pasa por evidenciar que la creciente asimetría entre China y Rusia no ha imposibilitado profundizar su “asociación estratégica". Un resultado favorecido por la mutua adaptación de China y Rusia a una relación asimétrica entre dos estados con identidad de gran potencia. Así, la dinámica de sus relaciones advierte un intercambio tácito por el que Rusia asume que es China quien determina la política económica y comercial de la integración euroasiática; mientras China se aclimata a las exigencias narrativas de Rusia para sostener su relato de liderazgo político en Eurasia.

Palabras clave: Relaciones chino-rusas. China. Rusia. Iniciativa de la Franjay la Ruta. Unión Económica Euroasiática.

\section{ABSTRACT}

This article analyses China-Russia relations since the launch of China's Beltand Road Initiative (BRI) regarding bilateral relations and the coordination of the BRI and the Eurasian Economic Union (EEU) led by Russia. This study draws three main conclusions. First, both Chinese and Russian leadership have made efforts to advance the relationship between their countries and to minimise the potential sources of conflict, which has led to real progress in the strategicasso- 
ciation reaching not only bilateral but also regional cooperation. Second, there is still a strong imbalance between the actual content of the agreements and the rhetoric employed, particularly in Russia, to project this relationship. Third, a growing asymmetry has not prevented the deepening of the strategic association. There has been a mutual adaptation to an asymmetric relationship. Russia has accepted that China determines the economic and commercial nature of the Eurasian integration, whereas China has adapted to Russian narratives and requirements to keep alive its role of political leadership in Eurasia.

Keywords: Sino-Russian Relations. China. Russia. Belt and Road Initiative. Eurasian Economic Union.

\section{Resumo}

Este artigo analisa as relações China-Rússia desde o lançamento da Iniciativa doCinturão eRota (ICR) daChina em relaçãoàs relações bilaterais eà coordenação do ICR e da União Econômica da Eurásia (EEU) liderada pela Rússia. Este estud o tira três conclusões principais. Em primeirolugar, tantoaliderança chinesa quanto a russa têm feito esforços para promover o relacionamento entre seus países e minimizar as potenciais fontes de conflito, o que levou a um progresso real na associação estratégica, al cançando não apenas a cooperação bilateral, mas também regional. Em segundo lugar, ainda há um forte desequilíbrio entre o conteúdo real dos acord os e a retórica empregada, especialmente na Rússia, para projetar essa relação. Terceiro, uma crescente assimetria não impediu o aprofundamento da associação estratégica. Houve uma adaptação mútua a uma relação assimétrica. A Rússia aceitou que a China determina a natureza econômica e comercial da integração da Eurásia, enquanto a China se adaptou às narrativas e aos requisitos russos para manter vivo seu papel de liderança política na Eurásia.

Palavras-chave: Relações sino-russas. China. Rússia. Iniciativa do Cinturão e Rota. União Econômica da Eurásia.

\section{Introducción}

En el debate sobre las relaciones entre China y Rusia, y en particular, acerca del alcance potencial de su "asociación estratégica", la obra de Bobo Lo (2008; 2017) y su concepto de "eje de conveniencia" es una inevitable referencia. El escepticismo del autor respecto a la naturaleza y sostenibilidad de la asociación estratégica entre las dos grandes potencias descansa en tres elementos: la existencia de intereses contrapuestos en el estricto ámbito bilateral, pero también entre los proyectos de integración regional liderados por China y Rusia; la divergencia en el diseño del futuro orden internacional llamado a sustituir el "momento unipolar" (KRAUTHAMMER, 1990) de supremacía estadounidense; y por último, la creciente asimetría entre China y Rusia en términos económicos, extensible, salvo en el terreno militar, al resto de capacidades estructurales.

Este artículo parte de una revisión del debate sobre las relaciones China-Rusia fundada en los tres aspectos arriba mencionados. A continuación, operando desde ellos, se analizan las relaciones China - Rusia desde el lanzamiento por China en 2013 de la Iniciativa de la Franja y la Ruta (IFR), tanto en lo respectivo a los acuerdos bilaterales, como en la coordinación de la IFR con la Unión Económica Euroasiática (UEE) en- 
cabezada por Rusia. Una etapa en las relaciones chino-rusas influida por el deterioro de las relaciones entre Rusia y Occidente a raíz de la crisis de Ucrania, el cual aceleró el previamente activado Pivot to Asia ruso. Este hecho, sin duda, afectó al enfoque del Kremlin, inicialmente escéptico, respecto al papel de Rusia en la IFR. No obstante, la crisis no solo ha provisto incentivos a Rusia para modelar su relación con China. "Ucrania" situó a Moscú ante la urgencia de sumar socios financieros y comerciales alternativos a Occidente, viéndose acuciada a adaptarse a las exigencias chinas en una relación económica asimétrica. Pero, en paralelo, el conflicto ha evidenciado que Rusia puede sostener en el tiempo una relación económica asimétrica, también la suya con Occidente lo es, pero no así la percepción de ser minusvalorada políticamente como actor regional y global hasta ver amenazada su seguridad. China es tras "Ucrania" más consciente de ello, produciéndose una mutua adaptación a la asimetría al compatibilizar la asertividad en defensa de sus intereses económicos, con la conformidad ante las condiciones impuestas por la derzhavnost rusa, esto es, su identidad de gran potencia.

En cuanto a la estructura de este artículo, tras la introducción se expone el debate académico sobre la naturaleza y alcance de las relaciones China-Rusia partiendo de una revisión crítica de la obra de Bobo Lo (2008; 2017). A continuación, en el tercer epígrafe, se analizan el origen y primer desarrollo de las relaciones China - Rusia. En el cuarto epígrafe, se examinan las relaciones entre China y Rusia desde el lanzamiento por China de la Iniciativa de la Franja y la Ruta (IFR) coincidente con la crisis de Ucrania, en lo respectivo a los acuerdos bilaterales. En el quinto, y último apartado previo a las conclusiones, se analiza la coordinación de la IFR con la Unión Económica Euroasiática (UEE) liderada por Rusia.

\section{El debate sobre la naturaleza y alcance de las relaciones entre China y Rusia}

Las relaciones entre China y Rusia, y en particular el alcance potencial del acercamiento en ellas, plantean un debate que cuenta ya con un desarrollo amplio en la literatura académica. Un punto de anclaje en el seno de ese debate, generador de múltiples discusiones posteriores, lo estableció Bobo Lo ya en 2008 con su concepto de "eje de conveniencia" [Axis of Convenience]. El autor exponía así su escepticismo respecto a la verdadera naturaleza y durabilidad de la asociación estratégica entre las dos grandes potencias (LO, 2008). Aun cuando el objetivo fuera refutarlas, tres nociones de la aportación neorrealista inicial de Lo han permanecido como ineludibles en su abordaje por los estudios sobre las relaciones chino-rusas. En primer lugar, Lo destacaba aspectos esenciales de la relación bilateral en los que Rusia y China divergían, tanto en términos de intereses - las consecuencias para la estrategia rusa en Asia-Pacífico de la creciente asertividad china o el choque de ambas potencias en Asia Central - o de coordinación en sus distintos proyectos de integración regionales donde se solapan zonas de influencia. Una oposición de carácter estructural que en opinión del autor dificultaría invertir el clima histórico de desconfianza en el que las relaciones entre China y Rusia se han desarrollado. 
La segunda de esas ideas fuerza es que la asociación se sustentaba en los beneficios que ofrece la cooperación en una coyuntura internacional específica: la de un orden internacional cuyas instituciones de gobernanza global retienen lo fundamental del diseño original otorgado por Occidente; y en el que la gestión delos Estados Unidos (EE.UU). de su supremacía, inclinada al unilateralismo, era percibida como una amenaza por ambas potencias. En la medida en que tanto para Rusia como para China los EE.UU. seguían siendo un socio preferencial, su propio acercamiento tenía cimientos frágiles. Además, diferían, según Lo, en el diseño del orden internacional llamado a sustituir lo que Krauthammer (1990) denominó el “momento unipolar". Según el autor, a pesar del discurso oficial chino y ruso en favor del multipolarismo, la expectativa de Vladímir Putin era tender hacia un "tripolarismo", mientras el punto focal de China no era otro que el bipolarismo. La última de esas tres ideas principales es la progresiva asimetría entre China y Rusia en términos económicos, extendida, salvo en lo militar, al resto de capacidades estructurales. Este desequilibrio más pronto que tarde afectará, en opinión de Lo, la estabilidad de la asociación estratégica producto de una previsible negativa de Rusia a contentarse con ser un "socio junior".

En posteriores trabajos el propio Bobo Lo ha mitigado el grado de escepticismo acerca del alcance de las relaciones entre China y Rusia. El autor reconoce el avance de importantes acuerdos de cooperación, si bien mantiene sus dudas, empleando el término de "asociación de conveniencia”, sobre la coincidencia de intereses a largo plazo más allá de contrarrestar la hegemonía de los EE.UU. y su influencia global (LO, 2017).

A continuación se desarrolla una síntesis del debate internacional, sin ánimo de exhaustividad, sobre las relaciones chino-rusas vertebrado en torno a los tres elementos adelantados por Lo (2008). En relación con la oposición de intereses bilaterales o regional-geopolíticos entre China y Rusia, permanecen vigentes enfoques que entienden que esas contradicciones antes o después supondrán un freno en la asociación estratégica entre China y Rusia (BUSZYNSKI, 2010; CARLSSON et al., 2016; FREEMAN, 2018; WATTS et al., 2016; YUJUN, 2014). El núcleo potencial de mayor tensión sigue siendo Asia Central, como un área en la que se solapan intereses y expectativas de influencia de ambas potencias. No obstante, algunos de los autores escépticos destacan elementos de avance en la cooperación, como Watts et al. (2016) en el ámbito militar, o Freeman (2018) en el de la energía.

Otro grupo de autores interpretan, por el contrario, que se tiende a exagerar las fuentes de rivalidad entre China y Rusia (DIESSEN, 2017). Entre ellos, sobresalen investigadores influyentes en sus respectivos países a nivel académico y político como Serguei Karaganov (2018), Alexander Lukin (2016a, 2016b, 2018) en Rusia; y Zhang Wenmu (2012) o Yan Xuetong (XUETONG, 2012, citado también en Kazak 2017 y Lukin 2018). Lukin (2018, p. 163-169) habla directamente de dos mitos en este sentido: el de la amenaza de la expansión demográfica china, en particular en el Lejano Oriente Ruso, y el de la competición por influencia en Asia Central. Aunque este grupo de autores también reconocen la existencia de terrenos de potencial desacuerdo, llaman a no confundir la competición 
económica, o las diferencias que no implican una amenaza, con la rivalidad geopolítica. Un tercer conjunto de especialistas, sin negar la presencia de áreas de eventual disenso en las que los intereses de Rusia y China difícilmente podrán coincidir, entiende que no comprometen per se la asociación estratégica ni la relevancia de su contenido (KACZMARSKI, 2015; KUHRT, 2014; 2018; WISHNICK, 2017).

En cuanto a las perspectivas rusa y china de evolución del orden internacional y de su estructura de gobernanza global, la hipótesis de Lo (2008) del "eje de conveniencia", o su versión actualizada de "asociación de conveniencia" (Lo, 2017), retiene el apoyo de parte de los especialistas (BUSZYNSKI, 2010; KEDI, 2016; WATTS et al., 2016). El propio Dmitri Trenin, director del Carnegie Moscow Center, y uno de los más reputados liberales rusos en el ámbito de las Relaciones Internacionales, exponía con anterioridad a la crisis de Ucrania argumentos en esta línea al entender que tanto Moscú como Pekín carecían de estrategias de largo término que pudieran mantener los vínculos requeridos por una relación estable (TRENIN, 2012).

Tras Ucrania, y a la luz de sus consecuencias y de las iniciativas de fortalecimiento de las relaciones chino-rusas los planteamientos de Trenin $(2015 ; 2018)$ cambiaron sustancialmente. En palabras del autor: "lo que fue originalmente un "matrimonio de conveniencia" con Pekín se ha convertido en una asociación mucho más estrecha que incluye cooperación en el comercio de energía, desarrollo de infraestructuras y defensa", si bien, "esto no presagia un nuevo bloque chino-ruso" (TRENIN, 2015, p. 1). La persistencia de la presión de los EE.UU. sobre ambas potencias, y en particular la vuelta de turca del pulso a China a partir de 2017 con su designación oficial como "rival estratégico", contribuyen a solidificar la asociación estratégica (TRENIN, 2018). Con los matices introducidos por cada autor, esos tres criterios son los predominantes en la literatura: la profundización en el contenido de la asociación estratégica, una visión común de la escena internacional y de sus amenazas por parte de Rusia y China, todo ello unido a la salvaguarda de ver improbable el ascenso de la relación al nivel de la alianza (BOLT; CROSS, 2018; BORDACHEV, 2016; CHASE et al., 2017; COX, 2016; GARVER, 2016; GELB; SIMES, 2013; LUKIN, 2018; ROZMAN, 2010; STOKES, 2017; WISHNICK, 2017). Autores como Rozman (2010), o Michael Cox (2016), alertan del error que puede suponer para los EE.UU. y la UE esperar que la hipótesis del "eje de conveniencia" se verifique. China y Rusia habrían adquirido "identidades paralelas" (ROZMAN, 2010) en la posguerra fría y hoy serían "dos potencias iliberales con ningún interés en sostener el actual orden liberal internacional" (COX, 2016, p. 1). Muchos de los estos especialistas hacen una referencia expresa al concepto acuñado por Lo (2008) para refutarlo, pero es Wishnick (2017) quien mejor condensa la nueva realidad al hablar de una "asociación de consecuencias", relevantes cabría añadir. Finalmente, aun cuando la anterior es la perspectiva más establecida, hay autores que consideran existen mimbres para alumbrar una alianza omnicomprensiva entre Rusia y China (DIESSEN, 2017; KARAGANOV, 2018; MARTYNUK, 2014; SUSLOV, 2018; XUETONG, 2012, citado en KAZAK, 2017 y en LUKIN 2018; WENMU, 2012). 
Respecto a la asimetría entre China y Rusia, y sus consecuencias, las aportaciones de los autores se pueden dividir en tres grupos. Uno primero se adhiere al escepticismo del autor australiano (BORDACHEV, 2017; BUSZYNSKI, 2010; CHASE et al., 2017; LARIN, 2014; WATTS et al., 2016; YUJUN, 2014).Algunos autores chinos, comoYujun(2014), considerana Rusia un lastrellamadoa limitar las decisiones tácticas másaudaces que China requiere para constituirse en una superpotencia global. No obstante, el argumento mayoritario acerca de por quéla asimetría puede debilitar la asociación entre ambas potencias afecta a Rusia. Una gran potencia que se está mostrando incapaz de frenar el cambio de énfasis chino en la asociación, desde lo bilateral a las iniciativas regionales y globales. Un escenario en el que a Rusia le cuesta no caer en un seguidismo donde la defensa de sus intereses se diluye (BORDACHEV, 2017; LARIN, 2014, p. 185). Siendo Rusia una gran potencia asertiva en la defensa de sus intereses, la asimetría contribuirá a debilitar la asociación.

En el otro extremo se posicionan varios autores rusos (KARAGANOV, 2018; LUKIN, 2016a, 2016b, 2018). Para ellos, entre Rusia y China no habría una asimetría de conjunto, a pesar de la superioridad económica china, ya que Rusia contrarrestaría ésta con su primacía militar y de influencia política global. Desde su perspectiva, en términos bilaterales, es prematuro situar el acento en la asimetría, pues lo correcto sería hablar de complementariedad, la cual además contribuye a cementar la asociación.

La tendencia más asentada en este debate reafirma la existencia de asimetría entre Rusia y China, pero subraya cómo la adaptabilidad a esa situación, principalmente de Moscú, pero asistido por gestos de Pekín, por el momento, no ha impedido profundizar en la asociación estratégica (BELLACQUA, 2010; KACZMARSKI, 2015; KUHRT, 2018; TRENIN, 2015; XUETONG, 2012; XUETONG citado en KAZAK 2012 y en LUKIN 2018; ZIEGLER, 2010). Cierto es que los postulados incorporan matices. Kaczmarski (2015), considera que, precisa mente, la asimetría impregna las relaciones chino-rusas y explica en buena medida su dinámica, así como alimenta el crecimiento de ésta. A mayor asimetría, mayor dependencia rusa (BELLACQUA, 2010; ZIEGLER, 2010), la cual, no obstante, ayuda a entender decisiones rusas para profundizar la asociación estratégica, no para disminuirla. Kuhrt (2018) se suma a esa corriente con cautela, advirtiendo que la asimetría puede provocar problemas a medio plazo si no se mantiene la voluntad de adaptación pragmática que se han demostrado mutuamente Rusia y China. Trenin (2015), por su parte, se distancia de Kaczmarski (2015) al afirmar contundentemente que Rusia no será dominada por China. A pesar de la asimetría, el Kremlin sabrá articular una "relación especial" con su socio de mutuo beneficio. El planteamiento de Yan Xuetong (2012; citado en Kazak, 2017, y en Lukin, 2018) es más particular, pues identifica la asimetría hasta el punto de defender una orientación bipolar del orden mundial, con los EE.UU. y China como únicas superpotencias, juzga que la asociación estratégica entre Rusia y China avanza a buen ritmo y debería escalar al grado de alianza.

Antes de cerrar el epígrafe conviene clarificar que existe una posición oficial de cada una de las dos grandes potencias para cada uno de estos debates exceptuando, por motivos obvios de prudencia, el de la asi- 
metría. Fu Ying, entonces viceministro de Asuntos Exteriores de China y hoy a cargo del Comité de Asuntos Exteriores de la Asamblea Popular Nacional de China, expuso a principios de 2016 en un artículo en Foreign Affairs la visión de China que, en lo esencial, sería aplicable a la rusa. El título con el que presentó su texto a las audiencias occidentales era explícito en cuanto a su intencionalidad: "How China Sees Russia: Beijing and Moscow are close, but not allies". El diplomático chino, rechazando de partida la idea de una alianza frente a Occidente, interpelaba directamente a Bobo Lo (2008; 2017) de la siguiente manera: "La relación chino-rusa es una asociación estratégica estable y de ningún modo un matrimonio de conveniencia: es compleja, robusta y fuertemente enraizada. Los cambios en las relaciones internacionales desde el fin de la Guerra Fría solo han acercado más a los dos países" (YING, 2016, p. 96).

\section{Origen y primer desarrollo de la "asociación estratégica" entre China y Rusia}

Entre 1992 y 1996, el enfoque de Andréi Kozyrev al frente del Ministerio de Asuntos Exteriores de Rusia se orientó hacia su integración en Occidente, lo cual conllevó en paralelo la consideración de China en un segundo plano. Un planteamiento con consecuencias tanto en la interpretación del nuevo orden internacional, por la minusvaloración de China en su condición de gran potencia, como en las relaciones bilaterales, desatendiendo la línea roja que para Pekín supone las relaciones entre terceros países y Taiwán (KUHRT, 2007, p. 9; LUKIN, 2018, p. 96-102). La llegada de Yevgeni Primakov al cargo de ministro de Asuntos Exteriores impulsó un enfoque "multivectorial" de política exterior. Esencia de la conocida como "Doctrina Primakov", el multivectorialismo suponía que Rusia, manteniendo como prioritarias las relaciones con Occidente, se abría a un acercamiento a los demás "polos de poder", dentro de una interpretación del sistema internacional ya entonces como multipolar. Ya en 1997, Boris Yeltsin y Jiang Zemin firmarían el documento fundacional de esa nueva relación: la "Declaración conjunta sobre un mundo multipolar y el establecimiento de un nuevo orden internacional". Una propuesta glosada de conceptos asociados con la versión westfaliana del Derecho Internacional - como los principios de respeto mutuo, soberanía e integridad territorial, no agresión, no injerencia en asuntos internos -, acompañados del énfasis ruso en la multipolaridad y los mantras chinos de mutuo beneficio y coexistencia pacífica (MORALES, 2017, p. 81).

El último periodo de Jiang Zemin como mandatario chino coincidió con la llegada de Putin a la presidencia rusa, y en él se firmaron nuevos acuerdos con la intención de, gradualmente, dotar de contenido al acercamiento chino-ruso y generar confianza mutua. Bajo esa perspectiva el documento base es el "Tratado de Buena Vecindad, Amistad y Cooperación" de julio de 2001, que establecía una "asociación equitativa basada en la confianza y la cooperación estratégica, limitada a ciertos asuntos de interés común" (MORALES, 2017, p. 81-82). El tratado, todavía vigente, proporcionó el cuerpo normativo de la cooperación bilateral e internacional sobre el que se ha construido la relación chino-rusa existente hoy. Pero si bien es cierto que el marco de asociación estratégica 
comenzó a tomar contenido, proyectando al mundo un compromiso para su estabilización, todavía permanece distante de las obligaciones y deberes que implican una alianza económica, política o militar.

Los años transcurridos entre el tratado de 2001 y el inicio de la "Gran recesión global" en 2008, fueron cursos tanto de un fuerte crecimiento económico como de un avance en el posicionamiento global de Rusia y China. En el ámbito internacional las dos grandes potencias protagonizaron conjuntamente los lanzamientos de la Organización para la Cooperación de Shanghái y del grupo de los BRICS. A pesar de que el apoyo ruso a los EE.UU. en Afganistán preocupó a China, la interacción chino-rusa se desarrolló en un marco de rechazo mutuo a la acción exterior de los EE.UU. - la invasión de Irak, las crisis nucleares en la península de Corea en 2002-2003 y 2009, o en las "Revoluciones de colores" en el espacio postsoviético (DE ANDRÉS; RUIZ-RAMAS, 2016) - . Asimismo, fue un periodo en el que, reteniendo siempre evidentes dosis de pragmatismo y búsqueda del beneficio directo para cada socio, se experimentó un marcado progreso en la cooperación bilateral. Se dieron pasos y acuerdos de calado como los que pusieron fin a las disputas fronterizas en el Lejano Oriente Ruso o la celebración de las primeras maniobras militares conjuntas. Sin embargo, especialmente destacó el incremento de iniciativas para fomentar el conocimiento y la confianza mutuas².

La "Gran recesión global" actuó como catalizador de lo que se puede considerar como un primer ensayo del PivottoAsia [PovorotkAzii] de Rusia. En 2009, los entonces presidentes Dmitri Medvedevy Hu Jintao se reunieron en seis ocasiones, firmándose nuevos acuerdos de cooperación energética y de seguridad - de especial calado el de septiembre de 2010 para "combatir el terrorismo, el separatismo y el extremismo"-. A pesar de ello, China no sobresalió en su asistencia financiera a Rusia, prefiriendo mantener los términos de cooperación económica en el intercambio comercial, y desviar en todo caso el diseño de alternativas financieras a los proyectos internacionales comunes como el grupo de los BRICS (STUENKEL, 2013).

Con Putin de nuevo en la presidencia, el lugary momento de presentar al mundo el Pivot to Asia no podía atesorar mayor simbolismo: el Foro de Cooperación Económica Asia-Pacífico (APEC por sus siglas en inglés) de 2012 celebrado en la principal ciudad del Lejano Oriente Ruso, Vladivostok. A solo dos meses de haber renovado Putin y Hu Jintao los términos de su asociación estratégica y un año después de que Barack Obama ejerciendo de anfitrión en la edición de 2011 en Honolulu expusiera el contenido de su Pivot to Asia. Vladivostok 2012 fue un acto con profundas aspiraciones performativas para situar a Rusia como potencia euroasiática con autonomía estratégica hacia EE.UU.y China y capacidad deliderazgo en Asia-Pacífico. Sin embargo, durante el siguienteaño, la retórica del Pivot to Asia ruso en Vladivostok no fue acompañada de manera acorde por nuevosproyectos de cooperación económica con los países de la APEC. La nueva política hacia Asia se mantuvo presente en los documentos y declaraciones que marcan la posición oficial rusa en materia de política exterior, comenzando por el más relevante de todos, el Concepto de Política Exterior aprobado en febrero de 2013. No obstante, en aquel
2. Sobresalen la celebración de diversos programas para dedicar un año de actividades para el conocimiento mutuo: 2004 fue el "Año de la amistad entre la juventud", 2006 el "Año de Rusia en China", 2007 el "Año de China en Rusia”, 2009 el "Año de la lengua rusa enChina, y2010 el “Año dela lengua china en Rusia”. 
periodo incluso perfiles cercanos al oficialismo del Kremlin y favorables al PivottoAsia ruso, como el de Fiodor Lukianov, reconocían que era todavía incierto y los pasos dados "un ritual, simbólicos" (LUKIANOV, 2013). La primera puesta en escena del PovorotkAzii evidenció, como nunca antes en las relaciones postsoviéticas entre Rusia y Asia-Pacífico, o más específicamente con China, el desequilibrio entre la pompa y las aspiraciones políticas frente a lo sustancial de los acuerdos alcanzados. Rompía a su vez con la concertación y coordinación entre Rusia y China de un cierto gradualismo en el ritmo de sus relaciones.Una dinámica de progresocontenido, en la que la promoción de la confianza mutua era en sí un valor, pero que dificultaba verificar o refutar de forma contundente las hipótesis esenciales en el debate académico sobre las relaciones ruso-chinas.

\section{Las relaciones y acuerdos bilaterales entre China y Rusia en la era del} IFR y la crisis de Ucrania

En otoño de 2013 coincidió la presentación oficial de la IFR con el inicio de las protestas del Maidán en Kiev que derivaron en la crisis de Ucrania, la cual ha acelerado la acción exterior rusa hacia Asia, y en particular su acercamiento a China. El deterioro de las relaciones con Occidente, la mutua imposición de sanciones, unido a los problemas de desarrollo y modernización de la economía rusa, alzaron la relevancia estratégica que Pekín poseía para Moscú. La primavera de 2014 marcó un punto sin retorno, vigente hasta la fecha, en las relaciones entre Occidente y Rusia. Tras la firma el 18 de marzo de los acuerdos de adhesión a la Federación de Rusia de la República de Crimea y la ciudad de Sebastopol, entre abril y junio acontecía la primera fase de la guerra del Donbass, un periodo de expansión de las milicias rebeldes apoyadas por Rusia en las regiones de Donetsk y Lugansk. En paralelo, junto a otros mecanismos de apoyo a las nuevas autoridades ucranianas, los EE.UU. y la UE respondían a la anexión de Crimea con la primera ronda de imposición de sanciones (RUIZ-RAMAS; MORALES, 2016, p. 127-155). En ese contexto, en los días 19 y 20 de mayo, Putin y Xi Jinping se reunieron en Shanghái en una cumbre previamente programada para tratar acuerdos bilaterales. El encuentro quedó fagocitado por la agenda internacional y Rusia lo presentó como el auténtico arranque del Pivot to Asia.

Rusiallegabaa lacumbre bilateral con la necesidad política de exhibir socios alternativos que acentuasen su independencia estratégica. Para lo cual debía dar salida a acuerdos relevantes de ámbito económico y comercial. La principal negociación, y la que marcaría el éxito o fracaso de la reunión, afectaba al sector energético. Un macrocontrato entre las principales empresas energéticas de cada país, Gazprom y China National Pretroleum Corporation (CNPC), ambas de titularidad pública, para suministrar gas natural ruso a China durante treinta años por valor de 400.000 millones de dólares. Tras una primera jornada marcada por el rechazo a las condiciones de la parte rusa, largamente negociadas en los años previos, China conseguía una reducción del precio del 14\%. Más importante aún es que China consiguió reproducir con Rusia, salvando igualmente reticencias de años, el modus operandi habitual de sus grandes 
corporaciones en proyectos desarrollistas en el extranjero: tomar el control de la construcción de las infraestructuras y que éstas se sufraguen con deuda china. En esta ocasión, unos 50.000 millones de dólares servirán para construir el gaseoducto principal y ejecutar los pozos de extracción en Siberia y el Lejano Oriente Ruso. Meses después, en febrero de 2015, el viceprimer ministro ruso Arkadi Dvorkovich anunció que Rusia seabría a la venta a compañíaschinas de activosenyacimientos estratégicos de gas y petróleo (GORDEYEV, 2015). Desde entonces se han producido distintas y relevantes operaciones, destacando el posicionamiento de CNPC en el desarrollo del gas natural licuado ruso, con apoyo financiero del China's Silk Road Fund en el proyecto Yamal LNG (FORBES, 2015), y del Banco de Desarrollo Chino en el futuro Arctic-LNG I y II (LNG WORLD NEWS, 2017). Un tipo de operaciones que hasta entonces Rusia había bloqueado a la participación de socios chinos. Para Rusia, la apertura a China del sector energético y de infraestructuras ruso ha sido una decisión dolorosa, y que en todo momento previo ha tratado de evitar. Muestra de ello son los documentos de Estrategia de Seguridad Nacional, que han definido recurrentemente como potenciales amenazas la persistencia del subdesarrollo de Siberia y el Lejano Oriente Ruso, la dependencia de las exportaciones de materias primas y la pérdida del control sobre sus recursos naturales.

Los acuerdos en el sector de la energía son útiles para identificar cinco patrones presentes en los acuerdos bilaterales entre China y Rusia en la era de la IFR tras la crisis de Ucrania. En primer lugar, en los sectores tradicionales de intercambio y cooperación comercial el ritmo de los acuerdos, y la envergadura de su contenido, se ha visto incrementado. Se ha hablado del gas, pero siendo las exportaciones de petróleo las principales en la cartera rusa hacia China, cabe destacar que, desde que en 2015 superase a Arabia Saudí, Rusia es el mayor proveedor de petróleo del mercado chino. Siendo cierto lo anterior, como segundo patrón, se ha de reiterar que mucha de la solemnidad comercial refiere en realidad a convenios no vinculantes, que incluyen directivas cuyos criterios de implementación son vagos y prolongados en el tiempo. En la cumbre de mayo de 2014se firmaron otros cuarenta y seis acuerdosy memorandos, si bien la mayor parte de ellos no eran vinculantes. Al margen del macrocontrato de gas "los resultados económicos de la cumbre fueron en el mejor de los casos modestos" (GABUEV, 2014).

En tercer lugar, en no pocos contratos de calado la urgencia rusa en la mesa de negociaciones se ha traducido en el traspaso de líneas rojas previas. En una coyuntura en la que los efectos de la asimetría entre estas potencias seven amplificados, Rusia ha accedido alos requerimientos sectoriales chinos. A los ejemplos en la industria de la energía se le suman varios en otro sector estratégico como es el de la venta de armas y equipamiento bélico. Tradicionalmente, dentro del mercado asiático, Rusia vendía su más avanzada tecnología exclusivamente a la India. Una política que conscientemente desplazaba a China del equipamiento de última generación, y defendida por Putin aun en 2013 (BLANK, 2013). Únicamente tras la crisis de Ucrania Rusia aceptó vender a China la tecnología más sofisticada, a pesar de temer por las consecuencias de que China 
pudiera aplicar ingeniería inversa y replicar sus avances. En el otoño de 2014 se firmó un contrato para vender a China sistemas de misiles tierra aire S-400 cuyo valor se estimó en 1.900 millones de dólares. En 2015, Rusia también accedió a la la entrega de veinticuatro cazas Sujoi Su-35 que se han ido entregando a partir de 2017. El cambio en la política de ventas es difícil de argumentar desde un enfoque puramente económico. El mercado asiático es casi tan nuclear para el sector armamentístico ruso, concentrándose allí el 70\% de su exportación; como el sector lo es para el mercado asiático, aglutinando un 43\% delas armasimportadas en la región (CONNOLLY; SENDSTAD, 2017, p. 11). Siendo así, los nuevos contratos no introducen otro elemento diferencial que soliviantar a otros socios tradicionales como, en particular, la India. La cesión rusa se comprende mejor si se integra en un marco de relaciones, con contraprestaciones cruzadas, en el que se quiere impulsar un salto cualitativo.

En relación con este último detalle figura el cuarto de los patrones presentes en los acuerdos bilaterales entre Rusia y China tras la crisis de Ucrania. Mientras China mantiene una estricta preocupación porloscontenidos y términos de cada acuerdo, Rusia asume concesiones contractuales, pero en compensación capitaliza en mayor medida la dimensión política del vigor en la asociación estratégica.

Como última pauta, se ha de constatar que, ni en la actualidad, ni en un plazo razonable que quepa recoger en un trabajo académico, cífrese en veinte años, China puede sustituir a la UE como principal socio comercial de Rusia, ni a Occidente en general como socio inversor, sin que los intereses rusos se vean intensamente dañados. Regresando al sector del gas natural, China solo recibe un $25 \%$ del volumen que Rusia suministra a la UE. Además, el proyectado crecimiento del suministro chino no implica que no ocurra lo propio en el suministro hacia la UE, donde se debate todavía el North Stream-2, y el porcentaje de gas ruso en el pool europeo se ha incrementado desde el 30\% al 37\% desde la anexión de Crimea. Con toda la infraestructura gasística apuntando hacia Europa, y contando el mercado asiático con más competidores, actores relevantes del sector ruso tienen claro que diversificar no es sinónimo de reorientar.

Siendo Rusia dependiente de sus exportaciones de materias primas, principalmente hidrocarburos, es lógico que lo que ocurre en el sector gasístico encuentre reflejo en las cifras del comercio en su conjunto. El volumen en euros de los flujos comerciales entre la UE y Rusia comenzaron un descenso acusado en 2012 y se mantuvieron hasta 2016. Es decir, comenzaron antes de la crisis de Ucrania y la imposición de sanciones y se han comenzado a revertir antes de que éstas se eliminen. En los cursos de 2017 y 2018 se ha dado un crecimiento sostenido de en torno al 20\% en las exportaciones rusas a la UE y de un 7.5\% en sentido inverso (THOMAS, 2018). Para la UE, Rusia sigue siendo el tercer destino mundial para sus exportaciones, pero la UE es el primero para Rusia. En el mismo periodo, China se ha consolidado como segundo socio comercial de Rusia solo por detrás de la UE. Pero la evolución del tráfico comercial no ha sido la proyectada en los memorandos firmados por sus gobernantes. Ya en junio de 2011, Hu Jintao y Medvedev acordaron en Moscú aumentar el comercio bilateral a 100.000 millones de dólares en 2015 y 200.000 en 2020. El pri- 
mer objetivo no se cumplió, e implicó que Putin y Xi volvieran a fijar el umbral de 100.000 millones como novedoso para diferentes anualidades. Según las estimaciones, la cifra psicológica se habría alcanzado en 2018, siendo prácticamente imposible doblar ese volumen en 2020. La aceleración del Pivot to Asia a causa de la crisis en Ucrania no habría tenido todavía un impacto apreciable en el comercio ruso-chino. La tendencia de crecimiento del comercio bilateral precisamente se estancó en 2014, cuyas cuotas solo se recuperaron en 2018 (LUKIN, 2018, p.142-145). Tanto en los flujos comerciales hacia la UE como hacia China, las causas de los altibajos son variadas, y salvo en sectores específicos, como las exportaciones alimentarias hacia Rusia, el impacto de las sanciones y el Povorot $k$ Azii son difíciles de medir. Se requieren análisis con una serie temporal mayor y que incluyan variables de control tales como las reacciones a las diversas crisis, globales o específicas, como la desaceleración china en 2015 o las devaluaciones del rublo; las variaciones en el precio de los hidrocarburos; la entrada de Rusia en la Organización Mundial del Comercio (OMC) en 2012, o la mayor presencia en el conjunto del comercio global de China, también por ejemplo en la UE.

Pero si había un sector que preocupaba a Rusia a consecuencia de las sanciones occidentales, y las posibilidades de encontrar sustitutos en los mercados internacionales, ese era el financiero. El cual es clave tanto en los flujos de asistencia financiera como en los de la inversión extranjera directa. Respecto a la inversión exterior china en Rusia, los analistas coinciden en que las cifras oficiales chinas y rusas no son fiables. Los datos del Banco de Rusia exceden a los de la Oficina Nacional de Estadísticas de China en varias anualidades hasta en veinte veces. La principal razón es que gran parte de la inversión china en Rusia proviene de capital que no está situado en China, sino en otras jurisdicciones fiscales, incluidos paraísos fiscales (KASHIN, 2017; LUKIN, 2018). Datos desagregados, recabados en las empresas chinas o por las administraciones regionales rusas indican que las inversiones desde 2014 han aumentado, teniendo en cuenta que se venía de niveles muy bajos, pero que se concentran en el sector de la energía y en sus infraestructuras. E incluso en esos sectores algunas de las inversiones anunciadas más suntuosamente se han quedado por el camino. Como la venta de un paquete de acciones de la petrolera Rosneft al conglomerado energético chino CEFC, o el tren de alta velocidad, denominado Eurasia para unir Pekín y Berlín a través de Moscú, cuya construcción se ha suspendido tras declararla infructífera (FINANZ.RU, 2018).

En cuanto a la asistencia financiera, los bancos chinos han estado muy lejos de proveer el crédito suficiente como para aliviar los efectos de las sanciones occidentales. Según Zhao Huaseng (2016), “Rusia presionó enfáticamente para obtener resultados rápidos, esperando el suministro de amplias cantidades de capital chino (...)Cuando los mercados rusos no recibieron el esperado volumen de capital chino, los expertos rusos vieron razones políticas tras ello". En opinión de Zhao no había motivo para ello, ya que sí se dio un incremento de las inversiones y crédito, pero los proyectos de gran envergadura tardan en proveer resultados. El autor chino recuerda que en la política exterior china la afinidad política tiene sus 
límites frente al pragmatismo económico: "no es realista esperar queChina invirtiera ciegamente grandes cantidades sin pensar en el beneficio".

En una síntesis sujeta a los debates expuestos en el segundo epígrafe, la crisis de Ucrania ha estimulado la cooperación bilateral económica entre Rusia y China, pero solo se puede hablar de un impacto notable cuantitativo y cualitativo en aquellos sectores en los que China posee mayor interés. Además, el contexto de la actual fase del PovorotkAzii ruso, caracterizado por la búsqueda de socios alternativos a 0ccidente por Moscú, ha impregnado los términos de los acuerdos. La sobrevenida necesidad rusa constituye, junto a la capacidad y modernización de sus economías, otro elemento de asimetría en la relación chino-rusa al debilitar su posición en la mesa de negociaciones.

La Iniciativa de la Franja y la Ruta y la Unión Económica Eurasiática: acomodarla asimetría

Xi Jinping anunció la IFR en el otoño de 2013, no por casualidad, en sendas visitas oficiales a Indonesia y Kazajstán. Pocos meses después, los presidentes de Rusia, Kazajstán y Bielorrusia firmaron el Tratado de la Unión Económica Eurasiática (UEE), el 29 de mayo de 2014, el cual entró en vigor el primero de enero de 2015. El escenario se prestaba a que los autores que planteaban que Rusia y China tenían intereses contrapuestos en Eurasia y Asia Central vieran sus hipótesis confirmadas. Atendiendo a los objetivos de las dos iniciativas de integración económica en Eurasia, la competencia chino-rusa podía ser de ardua conciliación. En mayo de 2015, durante una visita oficial de Xi a Putin, se presentó una declaración conjunta por la que se comunicaba la voluntad de combinar los proyectos, así como la creación de un mecanismo formal para su coordinación. Un año después, en el Foro Económico Internacional de San Petersburgo del 18 y 19 de junio, Putin se refería por vez primera a la propuesta rusa de "gran asociación Euroasiática", posteriormente conocida como la "Gran Eurasia”. La idea plantea la evolución de la coordinación entre la IFR y la UEE hacia una fórmula de integración euroasiática abanderada por Rusia. Un proyecto ambicioso al que le restan varios estadios de implementación para ser una realidad objetiva evaluable (RUIZ-RAMAS, 2020, p. 180-184); pero que, en cualquier caso, plantea un viraje vectorial de $\mathrm{Ru}$ sia desde la Gran Europa - Greater Europe, (MORALES, 2020; SAKWA, 2020) - al de la Gran Eurasia - Greater Eurasia - .

Aunque la IFR fue lanzada meses antes de la firma del Tratado de la Unión Económica Euroasiática, para entonces el proyecto liderado por Rusia estaba ya avanzado, con la Unión Aduanera Euroasiática entre Rusia, Kazajstán y Bielorrusia activa desde el 1 de enero de 2010. Más tarde se sumarían Kirguistán, Tayikistán y Armenia. El proyecto estandarte de la presidencia de Xi Jinping programa la construcción de infraestructuras para mejorar la conectividad de China con sus mercados y posee dos componentes principales: la "Franja Económica de la Ruta de la Seda" y la "Ruta Marítima del Siglo XXI". A ellas habría que añadir otros programas deámbito regional, entre los que aquí cabe destacar la llamada "Ruta de la Seda sobre hielo" que atravesaría el océano Ártico. El objetivo del 
diseño tanto de las rutas terrestres como de la marítima es alcanzar el continente europeo, pero son las dos ramificaciones principales terrestres las que incorporan a Eurasia como área geográfica esencial en la iniciativa. La IFR proyecta las prioridades y la preferencia de organización de las relaciones chinas en el espacio euroasiático: liderar la financiación y construcción de las infraestructuras de transporte; limitar las barreras al comercio, acomodarse o circunvalar potenciales proyectos de integracióny cooperación económica que pudieran obstaculizarsu penetración, así como bosquejar una formula replicable de cooperación con estados necesitados de estrategias desarrollistas. Aun cuando incorpora un mecanismo de financiación multilateral, el Banco Asiático de Inversión en Infraestructuras - junto a otro enteramente unilateral chino, el Fondo de la Ruta de la Seda -; la IFR funciona en lo institucional como un vehículo conducido unilateralmente por China en el que se desarrollan proyectos bilaterales entre China y cada uno de los Estados participantes. La IFR es tácticamente vaga en su definición y por tanto flexible para acoger como resultado propio cualquier acción bilateral china exterior tenga 0 no que ver con las comunicaciones. Pero sea como sea, a la capacidad de inversión y financiación de China va asociada una ganancia de influencia política de ámbito regional y bilateral. No obstante, la preocupación primera china es de índole económica: mantener la apertura de los mercados exteriores a su expansión económica que permita la exportación de su sobreproducción y exceso de capitales.

La celeridad con la que fue implementado el proyecto de integración euroasiático liderado por Rusia - con los establecimientos de la Unión Aduanera Euroasiática en 2010, el mercado único del Espacio Económico Euroasiático en 2012, y la UEE en 2015- evidencia una urgencia que traspasaba el marco de las necesidades de cooperación económica mutuas entre los países implicados. Hacia el oeste, Rusia se veía en la obligación de plantear una alternativa de integración a los estados de la Asociación Oriental, tentados por la UE para firmar respectivos Acuerdos de Asociación. Hacia el este, Rusia recelaba de la penetración económica de China en Asia Central, intranquilizando sobremanera el escenario de un proyecto regional chino que desafiase el liderazgo ruso en la Eurasia postsoviética. La UEE se ha consolidado en una importante estructura dentro de la política exterior rusa, no solo como un área de libre comercio sino como un bloque de relevancia geopolítica. No obstante, el progreso de la UEE se ha visto dificultado por disputas internas - desacuerdos sobre la normativa de comercio han derivado en breves guerras comerciales - y por un escaso impacto exterior al ser ignorada como un socio integral tanto por la UE como por las principales potencias asiáticas, incluida China (WOLCZUK; DRAGNEVA, 2017).

En dicho contexto, la primera reacción rusa al anuncio deXi Jinping en 2013 del lanzamiento de la IFR fue de cautela (GABUEV, 2017). La entrada masiva de inversiones y financiación, útil para obtener cambios normativos de apertura de mercados, amenazaba per se el ya frágil predominio económico ruso en Eurasia. Si además la relación entre la IFR y la UEE era una de competencia, el liderazgo político regional ruso quedaba desafiado en el medio plazo. La crisis de Ucrania y sus consecuencias, 
nuevamente, impulsaron un cambio en la predisposición del Kremlin hacia la participación de Rusia en la IFR. El 8 de mayo de 2015, Putin y Xi firmaban en Moscú la declaración política para "alinear" la IFR y la UEE - sopryazhenie, traducible también como conjugar o ligar-. El concepto posee mayor peso simbólico, apuntando hacia un nivel de relaciones superior a la mera cooperación, que contenido, dejando abiertos la forma y ritmo en que los acuerdos se desarrollarían.

En los dos primeros años de experiencia, de los cuarenta proyectos para mejorar las conexiones entre China y Europa planteados desde la UEE, casi todos por empresas rusas, Pekín no aceptó invertir en uno solo de ellos, argumentando modelos de financiación insostenibles y perspectivas de retorno imprecisas. Un problema de raíz es que no hay acuerdo sobre hacia qué dirección se deben dirigir esas infraestructuras de transporte. La atención de China se centra en el corredor este-oeste, mientras Rusia busca desarrollar el eje norte-sur desde el Lejano Oriente Ruso hacia Asia. En ese mismo periodo, entre 2015 y 2017, China mantuvo su insistencia en la liberalización de mercados de manera bilateral, pero también a través de la APEC, la OCS, así como en el marco de la nueva relación entre la IFR y la UEE. Rusia evitó posicionarse durante un tiempo. Finalmente, estimulados a su vez por el derrumbe del rublo, decidió entablar negociaciones en 2016 sobre una asociación económica y comercial integral con China en el marco de la UEE. Eso sí, Moscú integraba el acercamiento en un plan equivalente y que incorporaba a un total de doce estados (PANOV, 2016).

En paralelo, China ha seguido trabajando en infraestructuras de transportey energéticas en Uzbekistán, Kazajstány Tayikistán en el marco de la IFR pero producto de acuerdos bilaterales, siendo éste un riesgo identificado previamente por algunos autores rusos (BORDACHEV, 2015). De este modo, se puede concluir que el compromiso inicial para vincular la IFR y el UEE se sostuvo en mayor medida por sus implicaciones indirectas que sobre la base de los resultados directos. Moscú, necesitado de asistencia financiera y socios internacionales que contradijeran el relato del aislamiento y fortalecieran el del Povorot $k$ Azii, aceptaba participar en la IFR y asumía el rol proactivo de China en Eurasia. China, reconocía a la UEE como un socio equivalente en rango a su IFR, sin implicar modificar sus planes originales, a la que mantenía el pulso de su proyecto estrella, incorporando a una gran potencia a la IFR y asegurando su desarrollo pacífico en Eurasia.

\section{Conclusiones}

El estímulo de la asociación estratégica entre China y Rusia desde el otoño de 2013 y la primavera de 2014, periodos de impulso de la IFR y estallido de la crisis de Ucrania, es apreciable tanto en el contenido, con la firma de nuevos acuerdos e iniciativas de cooperación, como en la forma, con un salto cualitativo performativo en la proyección global de su acercamiento. Este cambio de ritmo espolea dinámicas latentes en las relaciones chino-rusas, lo que ha de servir para esclarecer las prioridadese intereses de ambas potencias en sus relaciones y ámbitos de cooperación. 
Un escenario de mayor fertilidad empírica en el que los argumentos e hipótesis presentes en el debate académico ganarán en precisión, alejándose de los elevados niveles de especulación que lo han caracterizado.

En lo concerniente a la proyección política global de la asociación estratégica, los últimos años respaldan las hipótesis que afirman su profundización, sin promocionar por ello esta relación al nivel de alianza. Ambas potencias han exhibido, en mayor comunión si cabe, su disenso con el orden internacional liberal bajo hegemonía estadounidense. Putin se encuentra en mejor sintonía con Xi, quien llegó al poder en 2013, al enfrentar el unilateralismo de los EE.UU., que esconde, bajo su perspectiva, una estrategia de "doble contención" verificable en la política de sanciones a Rusia y la reciente guerra comercial con China. Principios del Derecho Internacional asociados a la soberanía, como el de no injerencia, tienen una presencia constante en el vocabulario diplomático de Moscú y Pekín, percibiendo como una amenaza existencial los procesos de "cambio de régimen" auspiciados por Occidente.

Siendo esto así, se ha consolidado una disociación de prioridades, estrategias discursivas y estilos de policymaking en la política exterior de Rusia y China. Si China orienta el conjunto de su política exterior a concluir con éxito su proceso de desarrollo y modernización interior, Rusia dedica su experiencia internacional y sus mejores recursos a su reconocimiento como actor global, a asegurarse un lugar como gran potencia de ámbito global en el orden internacional que viene. En el terreno delos relatos, compartir el esfuerzo performativo de un orden multipolar implica para Rusia y China representar roles bien distintos. Mientras Rusia aspira con cada actuación a elevar su estatus global, China se esfuerza por contenerlo.InclusoXi, más asertivo que sus predecesores respecto ala posición internacional que ha de corresponder a China, se mantiene fiel a las narrativas delteatro multipolar. Finalmente, Rusiasemuestra más asertivay cómoda en el conflicto, reclamando presencia en todos aquellos foros en las que se trate una resolución de crisis internacional. Sin embargo, China fuera de su área de vecindad rehúye la confrontación y no le importa permanecer en un segundo plano, aun cuando posea intereses directos.

Las enmiendas compartidas por Rusia y China respecto al modelo de gobernanza global y sus distintas prioridades en política exterior favorecen que, a pesar de divergir en sus enfoques, se complementen en el terreno de la política internacional. Más complicado debiera ser identificar esta complementariedad de mutuo beneficio en elámbito económico. Mientras China es un entusiasta promotor de la globalización económica, si bien domesticada, Rusia aboga por un enfoque proteccionista basado en la substitución de importaciones. Hasta 2013, este antagonismo no había dificultado ni intervenido seriamente en la cooperación chino-rusa ni a nivel bilateral, ni en los proyectos de integración regional cuya membresía compartían. Pero la apuesta de Xi por liderar proyectos de expansión económica y comercial fuera de sus fronteras, en particular con la IFR, dificultaba a Rusia converger hacia espacios de complementariedad. China ha tendido a ofrecer concesiones a Rusia en la cooperación política, no importándole participar de la teatralización esbozada por Rusia de equivalencia entre las dos potencias. Pero se ha mantenido como 
un duro negociador para Rusia tanto en sus acuerdos comerciales bilaterales como en la conciliación de intereses en sus proyectos económicos exteriores. La experiencia vivida para compatibilizar la IFR con la Unión Económica Eurasiática confirma que China hace valer sus intereses económicos aprovechando la asimetría existente, mientras Rusia se adapta a esos intereses buscando capitalizar políticamente los pactos alcanzados, hasta ahora con la anuencia de Pekín.

Recapitulando, las principales conclusiones del estudio se sintetizan en tres argumentos. Por un lado, la voluntad de hacer progresar la asociación estratégica de gobernantes chinos y rusos, y en paralelo, de minimizar las fuentes de conflicto entre ambas potencias, han devenido en el avance sustancial de su cooperación. Una conclusión aplicable tanto a los acuerdos bilaterales como de integración regional. Por otro lado, siendo cierto lo anterior, persiste un fuerte desequilibrio entre el contenido real deestosacuerdosyla retórica conque, especialmente Rusia, proyecta sus relaciones con China. La tercera conclusión pasa por evidenciar que la asimetría entre China y Rusia, a pesar de mantenerse en aumento, no ha imposibilitado profundizar en la asociación estratégica. Un resultado favorecido por la mutua adaptación de China y Rusia a una relación asimétrica entre dos estados con identidad degran potencia. Así, la dinámica de sus relaciones advierte un intercambio tácito por el que Rusia asume que es China quien determina la política económica y comercial dela integración euroasiática; mientras China se aclimata a las exigencias narrativas de Rusia para sostener su relato de liderazgo político en Eurasia, el cual, a su vez, es condición necesaria para postularse como polo de un mundo multipolar. Una tolerancia a los anhelos geopolíticos rusos que no todos en China ven con buenos ojos. No olvidan que el relato ruso contiene una vocación performativa, esto es, que el actolingüístico produzca realidad.

\section{Referencias}

BELLACQUA, J. Introduction Contemporary Sino-Russian Relations: Thirteen Years of a 'Strategic Partnership. In: BELLACQUA, J. (ed.). The Future of China-Russia Relations. Lexington: University Press of Kentucky, 2010. p.1-13.

BLANK, S. The Context of Russo-Chinese Military Relations. American Foreign Policy Interests, v. 35 n. 5, p. 243-253, 2013.

BOLT, P. J.; CROSS, S. N. China, Russia, and Twenty-First Century Global Geopolitics. Oxford: Oxford Scholarship Online, 2018.

BORDACHEV, T. Novoe evrazistvo ["Nuevo eurasianismo"]. Russia in Global Affairs, n. 5, 14 oct. 2015. Disponible en: http://www.globalaffairs.ru/number/Novoe-evraziistvo-17754. Acceso en: 23 de noviembre de 2020.

BORDACHEV, T. Russia's Pivot to the East and Comprehensive European Partnership, Moscú: Valdai Discussion Club, 2016. Disponible en: https://valdaiclub.com/a/highlights/russia-pivot-to-the-east-and-comprehensive/ Acceso en: 23 nov. 2020.

BORDACHEV, T. ToRussia's Friends in Asia and Beyond, Moscow, Valdai Discussion Club, 2017. Disponible en: https://valdaiclub.com/a/highlights/to-russia-friends-in-asia-and-beyond/. Acceso en: 23 nov. 2020.

BUSZYNSKI, L. Overshadowed by China: The Russia-China Strategic Partnership in the Asia-Pacific Region. In: BELLACQUA, J. (ed.). The Future of China-Russia Relations. Lexington: University Press of Kentucky, 2010. p. 278-280.

CARLSSON, M.; OXENSTIERNA, S.; WEISSMANN, M. China and Russia - A Study on Coo- 
peration, Competition and Distrust. Ministry of Defense of Sweden Reports, report FOI-R--4087-SE, 2016.

CHASE, M. S.; MEDEIROS, E. S.; STAPLETON, J. R.; RUMER, E. B.; SUTTER, R.; WEITZ, R. Russia-china relations. Assessing Common Ground and Strategic Fault Lines. The National Bureau of Asian Research, Special report 66, 2017.

CONNOLLY, R.; SENDSTAD, C. Russia's Role as an Arms Exporter: The Strategic and Economic Importance of Arms Exports for Russia. Chatham House Research Paper, Londres, 2017.

Cox, M. Not just 'convenient': China and Russia's new strategic partnership in the age of geopolitics. Asian Journal of Comparative Politics, p. 1-18, 2016.

DE ANDRÉS SANZ, J.; RUIZ-RAMAS, R. Charles Tilly's concept of revolution and the "color revolutions". In: FUNES, M.J. (ed.). Regarding Tilly: Conflict, power and Collective Action. Nueva York: Rowman and Littlefield Publishing Group, 2016. p. 135-156.

DIESSEN, G. Russia's Geoeconomic Strategy for a Greater Eurasia. Londres: Routledge, 2017.

FINANZ.RU. Kitai obyavil nerentavelnym schel kovi put cherez Rossiu v Evropu ["China declara no rentable "ruta de la seda" a través de Rusia a Europa"]. Finanz-ru, 20 jun. 2018. Disponible en: https://www.finanz.ru/novosti/aktsii/kitay-obyavil-nerentabelnym-shelkovy-put-cherez-rossiyu-v-evropu-1027247658. Acceso en: 23 nov. 2020.

FORBES. Novatek prodal Fondu shelkovogo puti 9,9\% Yamal SPG [Novatek sold 9,9\% of Yamal LNG to the Silk Road Fund]. Forbes, 17 dic. 2015. Disponible en: http://www.forbes.ru/news/ 308637-novatek-prodal-fondu-shelkovogo-puti-99-yamal-spg. Acceso en: 23 nov. 2020.

FREEMAN, C. P. New strategies for an old rivalry? China-Russia relations in Central Asia after the energy boom. The Paciftc Review, v. 31, n. 5, p. 635-654, 2018. Disponible en: https://doi.or g/10.1080/09512748.2017.1398775. Acceso en: 23 nov. de 2020.

YING, F. How China Sees Russia: Beijing and Moscow are close, but not allies. Foreign Affairs, v. 95 n. 1, p. 96-105, 2016. Disponible en: https://www.foreignaffairs.com/articles/china/2015-12-14/how-china-sees-russia. Acceso en: 23 nov. 2020.

GABUEV, A. How Russia in Misreading Asia. Moscú: Carnegie Center Moscow, 2014. Disponible en: https://carnegie.ru/commentary/55776 Acceso en: 23 nov. 2020.

GABUEV, A. Bigger, Not Better; Russia Makes the SCO a Useless Club, Moscow Carnegie Center, jun. 23, 2017. Disponible en: http://carnegie.ru/commentary/71350 Acceso en: 23 nov. 2020.

GARVER, J. W. China's Quest: A History of the Foreign Relations of the People's Republic of China. Oxford: Oxford University Press, 2016.

GELB, L.; SIMES, D. A New Anti-American Axis?. Council on Foreign Relations, 6 jul. 2013. Disponible en: www.nytimes.com/2013/07/07/opinion/sunday/a-new-anti-american-axis.html. Acceso en: 23 nov. 2020.

GORDEYEV, V. Dvorkovich dopustil uchastie Kitaya v osvoenii strategicheskij mestorozhdeni ["Dvorkovich permitió la participación de China en el desarrollo de yacimientos estratégicos"]. RBK, Moscú, 27 feb. 2015. Disponible en: http://www.rbc.ru/economics/27/02/2015/ 54f002189a7947255e32ef80. Acceso en: 23 nov. 2020.

KACZMARSKI, M. Russia-China Relation in the Post-Crisis International Order. Nueva York: Routledge, 2015.

KARAGANOV, S. The new Cold War and the emerging Greater Eurasia. Journal of Eurasian Studies, v. 9, n. 2, p. 85-93, 2018. Disponible en: https://doi.org/10.1016/j.euras.2018.07.002. Acceso en: 23 nov. 2020.

KASHIN, V.Is China Investing Much in Russia?. Valdai Club, 9 jun. 2017. Disponible en : http:// valdaiclub.com/a/highlights/chinese-investments-in-russia/. Acceso en: 23 nov. 2020.

KAZAK, A. Time ripe for China and Russia to form an alliance - Chinese expert. RBTH, 17 mar. 2017. Accesible en: https://www.rbth.com/international/2017/03/17/china-russia-alliance-expert-721688. Acceso en: 23 nov. 2020.

KEDI,H. Russian Suspicions of China Hold BackTies. Global Times, 24 jul. 2016. Disponible en: http://www.globaltimes.cn/content/996163.shtml. Acceso en: 23 nov. 2020.

KRAUTHAMMER, C. The Unipolar Moment. Foreign Affairs: America and the World (1990/91), v. 70, n. 1, p. 23-33, 1990. Disponible en: https://www.foreignaffairs.com/articles/1990-01-01/unipolar-moment. Acceso en: 23 nov. 2020. 
KUHRT, N. Russian Policy Towards China and Japan: the Eltsin and Putin periods. Londres: Routledge, 2007.

KUHRT, N. 'Russia and China: strategic partnership or asymmetrical dependence?'. In: AKAHA, T., y VASILLIEVA, A. (eds.), Russia and East Asia: Informal and gradual integration, Londres y New York: Routledge, 2014. p. 91-108.

KUHRT, N. Asia-Pacific and China. In: TSYGANKOV, A. P. Routledge Handbook of Russian Foreign Policy. Londres: Routledge, 2018. p. 254-268.

LARIN, V. Russia and China: New Trends in Bilateral Relations and Political Cooperation. In: AZIZIAN, R.; LUKIN, A. (eds.). From APEC 2011 to APEC 2012: American and Russian Perspectives on Security and Cooperation in the Asia-Pacific. Honolulu, HI: Asia-Pacific Center for Security Studies; Vladivostok: Far Eastern Federal University Press, 2014. p. 178-189.

LNG WORLD NEWS. Novatek inks Arctic LNG 2 deals with Chinese partners. LNG World News, 1 nov. 2017. Accesible en: https://www.lngworldnews.com/novatek-inks-arctic-lng-2-deals-with-chinese-partners/. Acceso en: 23 nov. 2020.

LO, B. Axis of Convenience: Moscow, Beijing and the New Geopolitics. Londres: Royal Institute of International Affairs, 2008.

LO, B. A Wary Embrace: What the China-Russia Relationship Means for the World. Melbourne: Penguin and Lowy Institute, 2017.

LUKIANOV, F. My i novaya Azia [Nosotros y la nueva Asia]. Sovet po VneshneiiOvoronnoi Politike, 11 nov. 2013. Disponible en: http://svop.ru/ news/7661/. Acceso en: 23 de noviembre de 2020.

LUKIN, A. Russia in a Post-Bipolar Worl. Survival, v. 1, n. 58, p. 91-112, 2016 a.

LUKIN, A. Russia's Pivot to Asia: Myth or Reality?, Strategic Analysis, v. 6, n. 40, p. 573-589, 2016b.

LUKIN, A. China and Russia: The New Rapprochement. Cambridge: Polity Press, 2018.

MARTYNUK, V.Politicheskiy soyuz Rossii I Kitaya neizbezhen, potomu chto vygoden obeim strana, [La alianza política ruso-china es inevitable porque es en interés de los dos países]. Km.ru, 19 may. 2014. Disponible en: http://www.km.ru/world2014/05/19/vladimir-putin/740321'politicheskiisoyuz-rossii-i-kitaya-neizbezhen-potomu-chto-vy. Acceso en: 23 nov. 2020.

MORALES, J. Relaciones de Rusia con otras potencias en un mundo multipolar: Estados Unidos, Unión Europea y China. In: LÓPEZ JIMÉNEZ, J.Á.; MORALES HERNÁNDEZ, J., La política exterior de Rusia: Los conflictos y la construcción de un orden internacional multipolar. Madrid: Dykinson, S.L., 2017. p. 75-104.

MORALES, J. Identidades e intereses de Rusia hacia Europa: de la inclusión limitada a la (auto-) exclusión. In: RUIZ-RAMAS, R.; DE ANDRÉS, J.; MORALES, J. La Unión Europea y Rusia cara a cara: relaciones, conflictos e interdependencias. Valencia: Tirantlo Blanch, 2020. p. 27-60.

PANOV, P. Rossiya planiruet sozdat zoni svobodnoi torgovli s 12 stranami. Izvestia, 20 jun. de 2016. Disponible en: https://iz.ru/news/618319. Acceso en: 23 nov. 2020.

PUTIN, V. Plenary session of St Petersburg International Economic Forum. 17 jun. 2016. Disponible en: http://en.kremlin.ru/events/president/news/52178. Acceso en: 23 nov. 2020.

ROZMAN, G. The Sino-Russian Challenge to World Order: National Identities, Bilateral Relations, and East versus West in the 2010s. Stanford: Stanford University Press, 2010.

RUIZ-RAMAS, R. Del Greater Europeal Greater Eurasia: el Pivotto Asia de Rusia tras la crisis de Ucrania. In: RUIZ-RAMAS, R.; DE ANDRÉS, J.; MORALES, J. (eds.). La Unión Europea y Rusia cara a cara. Relaciones, conflictos e interdependencias. Valencia: Tirant lo Blanch. p. 165-190.

RUIZ-RAMAS, R.; MORALES, J. Un año y medio de guerra en el Donbass: de abril de 2014 a agosto de 2015.In: RUIZ-RAMAS, R (ed.). Ucrania. De la Revolución a la Guerra del Donbass. Salamanca: Comunicación Social, 2016.

SAKWA, R. Rusia, Ucrania y la UE entre dos modelos: monismo frente a pluralismo. In: RUIZ-RAMAS, R.; DE ANDRÉS, J.; MORALES, J. (eds.). La Unión Europea y Rusia cara a cara. Relaciones, conflictos e interdependencias. Valencia: Tirant lo Blanch, 2020. p. 108-122.

STOKES, J. Russia and China's Enduring Alliance: A Reverse' Nixon Strategy'Won'tWorkfor Trump. Foreign Affairs, 22 feb. 2017. Disponible en: https://www.foreignaffairs.com/articles/ china/2017-02-22/russia-and-chinas-enduring-alliance. Acceso en: 23 nov. 2020. 
STUENKEL, O. The Financial Crisis, Contested Legitimacy, and the Genesis of Intra-BRICS Cooperation. Global Governance, v. 4, n. 19, p. 611-630, 2013. Disponible en: https://doi. org/10.1163/19426720-01904008. Acceso en: 23 nov. 2020.

SUSLOV, D. The Limits of Global Containment: How to Win a New Cold War. Valdai Discussion Club, 2018. Disponible en: http://valdaiclub.com/a/ highlights/the-limits-of-global-containment/. Acceso en: 23 nov. 2020.

THOMAS, A. Russia's Trade With the West Surges Even as Sanctions Mount. The Wall Street Journal, 12 abr. 2018. Disponible en: https://www.wsj.com/articles/russias-trade-with-the-west-surges-even-as-sanctions-mount-1523534781. Acceso en: 23 nov. 2020.

TRENIN, D. True Partners? How Russia and China See Each Other. Londres: Centre for European Reform, 2012.

TRENIN, D. From Greater Europe to Greater Asia? The Sino-Russian Entente. Moscú: Carnegie Moscow Center, 2015. Disponible en: https://carnegie.ru/2015/04/09/from-greater-europe-to-greater-asia-sino-russian-entente-pub-59728. Acceso en: 23 nov. 2020.

TRENIN, D. Dynamism Hallmark of China-Russia Relations. Moscú: Carnegie Moscow Center, 2018. Disponible en: https://carnegie.ru/2018/03/19/dynamism-hallmark-of-china-russia-relations-pub-75821. Acceso en: 23 nov. 2020.

WATTS, J.; LEDBERG, S.; ENGELBREKT, K. Brothers in Arms, Yet again? Twenty-First Century Sino-Russian Strategic Collaboration in the Realm of Defence and Security. Defence Studies, v. 4, n. 16, p. 427-449, 2016. Disponible en: https://doi.org/10.1080/14702436.2016.1238747. Acceso en: 23 nov. 2020

WENMU. Z. ZhongE jiemengde xiandu mubiao he yiyi ["Los límites, objetivos y singnificados de formar una alianza chino-rusa"]. Guanchazhe, 5 mar. 2012. Disponible en: http://www. guancha.cn/ZhangWenMu/2012_03_05_66973.shtml. Acceso en: 23 nov. 2020.

WISHNICK, E. In search of the 'Other' in Asia: Russia-China relations revisited. The Paciftc Review, v. 1, n. 30, p. 114-132, 2017.

WOLCZUK, K.; DRAGNEVA, R. The Eurasian Economic Union: Deals, Rules and the Exercise of Power. Chatham House, 2 may. 2017. Accesible en: https://www.chathamhouse.org/2017/05/ eurasian-economic-union. Acceso en: 23 nov.2020.

XUETONG, Y. “Eluosi kekao ma? [¿Se puede confiar en Rusia?]. Guoji jingji pinglun(International Economic Review), n. 3,2012.

YUJUN, F. My view on Contemporary China-Russia Relations. Asan Forum, 25 jul. 2014. Disponible en: http://www.theasanforum.org/chinese-view/. Acceso en: 23 nov. 2020.

ZHAO, H., Zhong-E guanxi zhibianle ma? [¿Han cambiado las relaciones chino rusas cualitativamente?], Zhongguo xuanju yu zhili, Junio 6, 2016. Disponible en: http://www.chinaelections.com/article/778/242900.html. Acceso en: 23 nov. 2020.

ZHAO, H. Greater Eurasian Partnership: China's Perspective. China International Studies, $v$ 68, p. 68-84, 2018.

ZIEGLER, C. E. Russia and China in Central Asia. In: BELLACQUA, J. (ed.). The Future of China-Russia Relations. Lexington: University Press of Kentucky, 2010. p. 233-265. 